\title{
A three year prospective study of functional and clinical outcomes of total hip replacement at a tertiary care hospital
}

\author{
Addanki Vijayanand, Narreddy Jayasomeswar*
}

Department of Orthopaedics, Narayana Medical College and Hospital, Chinthareddypalem, Andhra Pradesh, India

Received: 02 January 2020

Accepted: 16 January 2020

\section{*Correspondence:}

Dr. Narreddy Jayasomeswar,

E-mail: sujithbhanu1212@gmail.com

Copyright: () the author(s), publisher and licensee Medip Academy. This is an open-access article distributed under the terms of the Creative Commons Attribution Non-Commercial License, which permits unrestricted non-commercial use, distribution, and reproduction in any medium, provided the original work is properly cited.

\begin{abstract}
Background: Hip joint is a crucial joint in the body whose function is essential for normal daily activities. Osteoarthritis of the hip cripples the daily functional capacity and total hip arthroplasty is considered to relieve the pain and increase the quality of life among these patients. The objective of present study was to assess the clinical and functional outcome in total hip replacement patients in terms of early joint function and stability of the hip joint and also to assess complications of procedures.

Methods: A three-year prospective study was conducted on 58 cases that fulfilled the inclusion criteria. Ethical approval and consent were obtained for the study. Standard clinical and laboratory evaluation was performed on all the cases and the data was noted in Microsoft excel sheet and analyzed. Modified Harris hip scoring was done pre operatively and postoperatively and follow-up was done at discharge, $4^{\text {th }}$ week, 6 months and one year.

Results: Surgery was performed on 58 cases with age range 34-73 years and a mean of 43.58 years. Avascular necrosis was the main indication (55.2\%). Maximum patient follow-up was 38 weeks. The mean preoperative score was 41.04 with minimum score being 10 and maximum being 76, postoperative score was 99.63 with minimum 64 and maximum was 109. Statistical significance was observed between preoperative and postoperative scores with regard to the parameters of pain, gait, functional activity and range of motion. Anterior thigh pain was the common postoperative complaint $(15.5 \%)$.

Conclusions: In conclusion, total hip replacement gives good clinical and functional outcomes. However, the outcomes are influenced by multiple factors which include indication for surgery, age of the cases, and type of prosthesis, operative technique and post-operative follow up.
\end{abstract}

Keywords: Hip joint, Osteoarthritis, Total hip replacement, Avascular necrosis

\section{INTRODUCTION}

Normal hip joint is a crucial joint in the body which bears daily stress and strains due to various activities performed in daily life. Its physiological functioning is essential for normal and peaceful day to day life. With a change in life style, dietary habits the incidence of chronic disabling conditions like osteoarthritis; inflammatory arthritis and osteonecrosis are on the rise and incapacitating the daily functions. ${ }^{1}$ With the increasing age the common condition associated with the hip is osteoarthritis which cripples the daily functional capacity. In this clinical condition, total hip arthroplasty (THR) is considered a best choice of procedure for bringing back the regular daily activities. THR is one of the common orthopaedic reconstructive surgeries which have relieved millions of people living with crippling pain and deformity arising from the hip joint. ${ }^{2}$ THR was first performed by Philip Wiles in 1938 and later on with several modifications in the original procedure by Sir John Charley and others. Improvements in implant design materials and fixation techniques continued but Charnely's basic concept is still 
valid. This is a highly cost-effective procedure which definitely improves the quality of life. Several factors contribute in development of complications post THR and eventually may result in failure of the procedure which may be poor selection of the patients, materials and design of the implant. To determine the durability of THR, evaluation of long-term outcomes is important. Patient derived outcome scales are important to surgeons for measuring improvement in surgical techniques and interventions based on the indications of surgery. The Harris hip score is the most widely used scoring system for evaluating hip arthroplasty. ${ }^{3}$

The aim of this study was to assess the clinical and functional outcome in THR patients in terms of early joint function and stability of the hip joint and also to assess complications of procedures.

\section{METHODS}

The present prospective cross-sectional study was conducted by Department of orthopaedics at Narayana Medical College a tertiary care hospital in south India for a period of three year from January 2016 to December 2018. The study protocol was presented before the institutional ethical committee and approved. The study was conducted as per the guidelines of the ethical committee. The study participants were informed about the study protocol and written informed consent was obtained from all the cases. A thorough history and clinical examination was done and data was noted in a separate predesigned questionnaire sheet. Preoperative laboratory and radiological investigations were performed and the findings were noted. On clinical examination the sensory motor examination, vascularity of the limb, deformities of the hip, range of motion (ROM) of the hip and other joints were noted. Deformity and ROM were measured by goniometer. All the patients were assessed using Modified Harris hip score preoperatively and post-operatively. All patients were operated with common standard postero lateral approach.

\section{Inclusion criteria}

All the patients who underwent uncemented total hip replacement for isolated hip pathologies.

\section{Exclusion criteria}

Patients who had undergone uncemented total hip replacement with deformities or pathologies of other joints of lower limb which may affect the functional outcome.

\section{Postoperative follow-up}

Prophylactic antibiotics were administered 24 hours prior and continued until 72 hours postoperatively and shifted to oral form in 7 days. Deep venous thrombosis (DVT) prophylaxis in the form of heparin was administered for five days postoperatively. Drains were removed within 24-48 hours of surgery. On first post op day, check Xrays were taken, patient was taught quadriceps exercises, knee and ankle mobilization and made to sit. Second post op day, gait walking with walker was taught with weight bearing to tolerance. Sutures were removed on $12-14^{\text {th }}$ day and discharged from hospital. Modified Harris hip scoring was done at discharge, $4^{\text {th }}$ week, 6 months and one-year follow-up. Based on a total of 100 points, a score of 90-100 is reported as excellent results, 80-90 being good, 70-79 fair, 60-69 poor and below 60 a failed result.

\section{Statistical analysis}

All the collected data was entered in Microsoft excel spread sheet and analysed by SPSS version 22 (Chicago, USA). The results were averaged (mean \pm standard deviation) for each parameter for continuous data and numbers and percentage for categorical data. $\mathrm{P}$ value $<0.01$ was considered statistically significant in the study.

\section{RESULTS}

The present prospective study was carried out for a period of three years with a total of 58 cases who fulfilled the inclusion criteria. Male predominance was observed in our study with $38(65.5 \%)$ cases and females 20 cases $9(34.5 \%)$. The age range of participants in the study was 34-73 years with a mean age of 43.58 years at the time of surgery. Majority of the cases $(44.8 \%)$ were in the age group of 31-50 years followed in order by $>50$ years $(41.4 \%)$ and $<30$ years $(13.8 \%) .58 .6 \%$ of cases were operated on the right and $41.4 \%$ on the left. The most common indication for surgery was avascular necrosis $(55.2 \%)$ followed by osteoarthritis $(27.6 \%)$, ankylosing spondylitis (10.3\%) and rheumatoid arthritis (6.9\%). (Table 1).

Ten cases were followed up till six months and 28 cases (48.28\%) were followed between $>6-24$ months and 20 cases were followed $>24$ months. The maximum patient follow-up was up to 38 weeks in the study (Table 2).

Modified Harris hip score was used to evaluate the functional outcome of cases in our present study. For the total score and each of the parameters in the score, higher score implies lesser disability. The mean total preoperative score was 41.04 with minimum score being 10 and maximum being 76 . The postoperative score was 99.63 with minimum 64 and maximum was 109 . In the present study, $\mathrm{p}$ value of less than 0.05 implies statistical significance. In the present study, statistical significance was observed between preoperative and postoperative scores with regard to the parameters of pain, gait, functional activity and ROM and absence of significance was observed with regard to post-operative deformity indicating that no major deformities were observed postoperatively in majority of the cases (Table 3 ). 
Table 4 summarizes the comparison of the preoperative vs post-operative scores according to grading and reported as 90-100 for excellent results, 80-90 being good, 70-79 being fair, 60-69 poor and less than 60 a failed result. Preoperatively $75.86 \%$ had poor score, $20.69 \%$ had fair score. Postoperatively the result showed, $86.21 \%$ had excellent score, $10.34 \%$ good and only two cases had a fair result. In the study, all the cases with $<50$ years had excellent postoperative outcome scores and all the cases with good and fair outcome was above 60 years of age and no statistical significance was observed in the study with relation to age and outcome.
Anterior thigh pain was the common postoperative complaint $(15.5 \%)$ and other less common were stress shielding (10.3\%), osteolysis (6.9\%), superficial infection $(6.9 \%)$, periprosthetic fracture $(6.9 \%)$ and Nerve injury (3.4\%). $75.9 \%$ of cases in the study had no postoperative complications (Table 5). Two cases that developed periprosthetic fracture had a fair outcome in our study, and of the six cases with good outcome, two had nerve injury, two had anterior thigh pain and two had stress yielding fracture.

Table 1: Age group, sex and indications for THR among the cases.

\begin{tabular}{|lll|}
\hline Variable & N & $\%$ \\
\hline Gender & & 65.5 \\
\hline Male & 38 & 34.5 \\
\hline female & 20 & \\
\hline Age group (years) & & 13.8 \\
\hline$<30$ & 8 & 44.8 \\
\hline $31-50$ & 26 & 41.4 \\
\hline$>50$ & 24 & 55.2 \\
\hline Indications for THR & & 81.25 \\
\hline Avascular necrosis & 32 & 18.75 \\
\hline Idiopathic & 26 & 27.6 \\
\hline Post traumatic & 6 & 10.3 \\
\hline Osteoarthritis & 16 & 6.9 \\
\hline Ankylosing spondylitis & 6 & \\
\hline Rheumatoid arthritis & 4 & 58.6 \\
\hline Side of hip arthroplasty & & 41.4 \\
\hline Right & 34 & \\
\hline Left & 24 & \\
\hline
\end{tabular}

Table 2: Duration of follow-up of cases in the study.

\begin{tabular}{|lll|}
\hline Follow up months & No & $\%$ \\
\hline $\mathbf{6}$ & 10 & 17.24 \\
\hline$>\mathbf{6 - 2 4}$ & 28 & 48.28 \\
\hline$>\mathbf{2 4}$ & 20 & 34.48 \\
\hline
\end{tabular}

Table 3: Preoperative and postoperative scores according to various parameters of modified Harris hip scoring system.

\begin{tabular}{|c|c|c|c|c|c|c|c|}
\hline & & $\mathbf{N}$ & Mean & SD & Min & Max & $P$ value \\
\hline \multirow{2}{*}{ Pain } & Pre-operative & 58 & 14.65 & 3.42 & 10 & 30 & \multirow{2}{*}{$<0.001$} \\
\hline & Post-operative & 58 & 44.25 & 4.21 & 35 & 48 & \\
\hline \multirow{2}{*}{ Function-gait } & Pre-operative & 58 & 12.37 & 8.54 & 0 & 25 & \multirow{2}{*}{$<0.001$} \\
\hline & Post-operative & 58 & 28.29 & 3.59 & 11 & 36 & \\
\hline \multirow{2}{*}{ Function-activity } & Pre-operative & 58 & 6.72 & 2.89 & 0 & 12 & \multirow{2}{*}{$<0.001$} \\
\hline & Post-operative & 58 & 18.26 & 1.23 & 10 & 15 & \\
\hline \multirow{2}{*}{$\begin{array}{l}\text { Absence of } \\
\text { deformity }\end{array}$} & Pre-operative & 58 & 4.32 & 1.64 & 0 & 5 & \multirow{2}{*}{0.168} \\
\hline & Post-operative & 58 & 5.24 & 0.41 & 4 & 5 & \\
\hline \multirow{2}{*}{ ROM score } & Pre-operative & 58 & 2.98 & 1.48 & 0 & 4 & \multirow{2}{*}{$<0.001$} \\
\hline & Post-operative & 58 & 3.59 & 0.24 & 4 & 5 & \\
\hline \multirow{2}{*}{ Total score } & Pre-operative & 58 & 41.04 & 17.97 & 10 & 76 & \multirow{2}{*}{$<0.001$} \\
\hline & Post-operative & 58 & 99.63 & 9.68 & 64 & 109 & \\
\hline
\end{tabular}


Table 4: Comparison of the pre-operative vs post-operative scores according to grading $(\mathbf{n}=\mathbf{5 8})$.

\begin{tabular}{|lll|}
\hline Outcome score (grade) & Pre-operative & Post-operative \\
\hline Poor & $\mathbf{N}(\boldsymbol{\%})$ & N $(\mathbf{\%})$ \\
\hline Fair & $44(75.86)$ & 0 \\
\hline Good & $12(20.69)$ & $2(3.45)$ \\
\hline Excellent & 0 & $6(10.34)$ \\
\hline
\end{tabular}

Table 5: Frequency of various complications.

\begin{tabular}{|lll|}
\hline Complications & N & $\%$ \\
\hline Nerve injury & 2 & 3.4 \\
\hline Superficial infection & 4 & 6.9 \\
\hline Periprosthetic fracture & 4 & 6.9 \\
\hline Anterior thigh pain & 9 & 15.5 \\
\hline Stress shielding & 6 & 10.3 \\
\hline Osteolysis & 4 & 6.9 \\
\hline No complication & 44 & 75.9 \\
\hline
\end{tabular}

\section{DISCUSSION}

Total hip replacement is a well-documented surgical procedure which improves the quality of life of the patients when properly performed and managed. Male preponderance was observed in our study with $65.5 \%$ of cases which explains that males are more prone to trauma and avascular necrosis which is a common indication for replacement of the hip. $45 \%$ of the cases in the study were in the age group of 31-50 years with mean age of 43.58 years and age range of 34-73 years which is on par with findings of Sandesh et al who reported that $50 \%$ of cases below age of 50 years and age range of 34-82 years. ${ }^{4}$ Avascular necrosis was the most common indication for replacement $(55.2 \%)$ with osteoarthritis next common (27.6\%). Majority of the studies universally state that avascular necrosis as the most common cause of replacement. ${ }^{5}$ Few studies reported arthritis secondary to avascular necrosis as the most common cause which is contrary to the findings of our study. ${ }^{6}$

Modified Harris score was used to evaluate the functional outcome of the cases in the present study. In our study, excellent results were obtained in $86.21 \%$ of the cases and good in $10.34 \%$ of cases after total hip arthroplasty. The mean total preoperative score was 41.04 which improved postoperatively to 99.63 . The results of our study were comparable with the findings in the study of Siwach et al who performed THR on 128 cases with $87 \%$ of excellent results in his study and Chandrasekhar et al who reported the same results in his study. ${ }^{7,8}$ In the present study, statistical significance was observed between preoperative and postoperative scores with regard to pain, gait, functional activity and range of motion. Similar results were observed with the findings of Siavashi et al who reported significance with same parameters in our study. ${ }^{9}$ However few studies reported significance in pain, gait only and non-significance in ROM and post-operative deformity. ${ }^{10}$ In the present study, all the patients with age less than 50 years had excellent results and between 60-75 years had good and fair results which are on par with the results of Espehaug et al who reported favourable results of total hip replacements with non-inflammatory osteoarthritis less than 45 years old. ${ }^{11}$ There was no statistical significance associated with age and result score in our study.

Anterior thigh pain was the common complication $(15.5 \%)$ in our study, which was similarly reported in the findings of Weber et al with $68 \%$ in his study. ${ }^{12}$ Stress shielding was another common complaint observed in $10.3 \%$ of cases; few studies reported periprosthetic fracture as the major complication which was not observed in our study. Superficial infection was observed in $6.9 \%$ of the cases in our study and was managed with long duration antibiotic administration and delayed suture removal. Superficial infection was reported among $9 \%$ of the THR cases in the study of Dale et al. ${ }^{13}$ However the incidence of post-operative infections are variable from place to place depending upon multiple factors.

In conclusion, total hip replacement gives good clinical and functional outcomes. However, the outcomes are influenced by multiple factors which include indication for surgery, age of the cases, and type of prosthesis, operative technique and post-operative follow up. Better analysis can be made if long term studies are done and follow up for a long period. In our study, the overall functional and clinical outcome demonstrated.

Funding: No funding sources

Conflict of interest: None declared

Ethical approval: The study was approved by the institutional ethics committee

\section{REFERENCES}

1. Shan L, Shan B, Graham D, Saxena A. Total hip replacement: a systematic review and meta-analysis 
on mid-term quality of life. Osteoarthritis Cartilage. 2014;22(3):389-406.

2. Crawford RW, Murray DW. Total hip replacement: indications for surgery and risk factors for failure. Annal Rheumatic Dis. 1997;56:455-7.

3. Smith GH, Johnson S, Ballantyne JA, Dunstan E, Brenkel IJ. Predictors of excellent early outcome after total hip arthroplasty. J Orthop Surg Res. 2012;7(13):66-71.

4. Sandesh YR, Ram GG, Kunal P, Giriraj H, Phagal VV. Functional and radiological outcomes of total hip replacement in non traumatic indications. Int $\mathbf{J}$ Curr Microbiol App Sci. 2014;3(3):153-8.

5. Barrack RL, Mulroy RDJ, Harris WH. Improved cementing techniques and femoral component loosening in young patients with hip arthroplasty. A 12-year radiographic review. J Bone Joint Surg Br. 1992;74:385-9.

6. Kim YH. Total hip arthroplasty in adult patients who had childhood infection of the hip. J Bone Joint Surg Am. 2003;85:198.

7. RC Siwach, Kadyan VS, Sangwan SS, Gupta R. A retrospective study of total hip arthroplastyIndian J Orthop. 2007;41(1):62-6.

8. Sekhar C, Mittal A, Rallapalli R, Biju R, Prasad YS. Evaluation and Outcome of Total Hip Replacement in Adults with Arthritis. IOSR-JDMS. 2015;14(4):65-72.
9. Siavashi B, Mohseni N, Zehtab MJ, Ramim T. Clinical Outcomes of Total Hip Arthroplasty in Patients with Ankylosed Hip. Arch Bone Joint Surg. 2014;2(1):25-30.

10. Luo Y, Yang Z, Yeersheng R, Li D, Kang P. Clinical outcomes and quality of life after total hip arthroplasty in adult patients with a history of infection of the hip in childhood: a mid-term followup study. J Orthop Surg Res. 2019:14:38.

11. Espehaug B, Havelin LI, Engesaeter LB, Langeland $\mathrm{N}$, Vollset SE. Patient satisfaction and function after primary and revision total hip replacement. Clin Orthop Relat Res. 1998;351:135-48.

12. Weber M, Craiovan B, Woerner ML, Schwarz T, Grifka J, Renkawitz TF. Predictors of Outcome After Primary Total Joint Replacement. J Arthroplasty. 2018;33:431-5.

13. Dale H, Skråmm I, Løwer HL, Eriksen HM, Espehaug B, Furnes O, Engesaeter LB. Infection after primary hip arthroplasty: a comparison of 3 Norwegian health registers. Acta Orthopaedica. 2011;82(6):646-54.

Cite this article as: Vijayanand A, Jayasomeswar N. A three-year prospective study of functional and clinical outcomes of total hip replacement at a tertiary care hospital. Int J Res Orthop 2020;6:308-12. 